\title{
Growth of Pseudomonas Species on Phenylacetamide
}

\author{
By JOAN L. BETZ AND PATRICIA H. CLARKE \\ Department of Biochemistry, University College London, \\ Gower Street, London, WCI $E 6 B T$
}

(Received 12 October 1972)

\begin{abstract}
SUM M A R Y
Wild-type strains of Pseudomonas putida, $P$. cepacia and $P$. acidovorans grew in minimal salts media with acetamide or phenylacetamide as the carbon or nitrogen source. Wild-type strains of $P$. aeruginosa utilized acetamide but not phenylacetamide. Pseudomonas putida A90 and mutants isolated from $P$. putida A87 and from $P$. cepacia $7 \mathrm{I} 6$ grew on phenylacetamide but not on acetamide. These activities were due to two types of amidase : (i) aliphatic amidases, which hydrolyse acetamide, produced by $P$. aeruginosa and strains of $P$. putida, $P$. cepacia and $P$. acidovorans; and (ii) phenylacetamidases, which hydrolyse phenylacetamide, produced by some strains of $P$. putida, $P$. cepacia and $P$. acidovorans.
\end{abstract}

\section{INTRODUCTION}

Stanier, Palleroni \& Doudoroff ( 1966 ) found that several species of aerobic pseudomonads grew on acetamide as carbon source. All the strains of Pseudomonas aeruginosa tested by Clarke (1972) grew on acetamide; some strains of $P$. putida biotype A and all of the strains of $P$. acidovorans and $P$. cepacia which were examined also grew on acetamide. The aliphatic amidases produced by these species were very similar in substrate specificities, with optimal activities for acetamide and propionamide, but there were differences in the range of amides which these species could utilize for growth. Some strains of $P$. putida A and $P$. acidovorans grew on butyramide and this was due to the induction of an aliphatic amidase by butyramide. Amidase was not induced by butyramide in any of the $P$. aeruginosa wild-type strains, but some mutants which synthesize the wild-type enzyme grew constitutively on butyramide (Brown \& Clarke, 1970). Pseudomonas cepacia strains did not grow on butyramide and acetamidase was not induced when butyramide was added to exponentially growing cultures (Clarke, 1972).

Pseudomonas putida, P. acidovorans and P. cepacia metabolize phenylacetate and use it as a carbon source for growth although $P$. aeruginosa is unable to do so (Stanier et al. 1966). We have isolated mutants of $P$. aeruginos $a$ which hydrolyse phenylacetamide and utilize it as a nitrogen source (but not carbon source) for growth (Betz \& Clarke, I972). These mutants produce amidases which have altered substrate specificities such that the enzymes have little or no activity for acetamide hydrolysis. We found that $P$. acidovorans, $P$. cepacia and all the strains of $P$. putida A which were tested, including those unable to grow on acetamide, utilized phenylacetamide as both carbon and nitrogen source. Since this appeared to require the hydrolysis of phenylacetamide we have compared the naturally occurring phenylacetamidases of $P$. putida A, $P$. acidororans and $P$. cepacia with the phenylacetamidases derived in the $P$. aeruginosa mutants by selective evolution in the laboratory. 


\section{METHODS}

Bacteria. The strains used are listed in Table I. We are grateful to Professor R. Y. Stanier for providing the strains of Pseudomonas putida and P. cepacia (from the Berkeley collection). Pseudomonas acidovorans NCIB 968I was obtained from the National Collection of Industrial Bacteria. The constitutive mutant of $P$. putida A87 was isolated by the method of Brammar, Clarke \& Skinner (1967). The acetamide-negative mutants of $P$. putida $\mathrm{A} 87$ and $P$. cepacia 716 were isolated as fluoroacetamide-resistant mutants (Clarke \& Tata, 1973).

Growth of cultures. Cultures were maintained on nutrient agar slopes. Minimal salts medium (Brammar \& Clarke, 1964) was used for all growth experiments with the addition of various compounds as carbon and nitrogen sources. Succinate, pyruvate, lactate (as their sodium salts) and phenylacetamide were added before autoclaving. Other amides were sterilized by filtration and added aseptically to the minimal salts medium. Solid media contained $\mathrm{I} \cdot 0 \%(\mathrm{w} / \mathrm{v})$ Oxoid agar no. I. Plate media contained as carbon or nitrogen sources the following compounds $(\%, w / v)$ : acetamide $(\mathrm{AM}), 0.3$; valeramide $(\mathrm{V}), 0.2$; hexanoamide $(\mathrm{H})$, $0 \cdot \mathrm{I}$; succinate and phenylacetamide $(\mathrm{S} / \mathrm{Ph}), \mathrm{I} \cdot 0$ and $0 \cdot \mathrm{I}$; pyruvate and phenylacetamide (Pyr/Ph), 0.5 and 0.I. Growth tests were as described by Betz \& Clarke (1972).

Enzyme synthesis during growth. Cultures were grown overnight in $5 \mathrm{ml}$ of medium in $25 \mathrm{ml}$ universal screw-cap bottles. Measurements on enzyme synthesis during exponential growth were carried out with $100 \mathrm{ml}$ medium in I l conical flasks incubated on a shaker, at $37^{\circ} \mathrm{C}$ for Pseudomonas aeruginosa and $30^{\circ} \mathrm{C}$ for the other strains. Samples were kept on ice until amidase and amide assays could be carried out.

Measurement of bacterial growth. Bacterial growth was estimated by measuring the extinction at $670 \mathrm{~nm}$ with a Unicam SP 600 spectrophotomer. The growth rates varied widely in the different media used and the spectrophotometer readings were used directly and not converted into dry weights.

Enzyme assays. Transferase assays were carried out by the method devised by Brammar \& Clarke (1964) with the modifications introduced by Betz \& Clarke (I972) for assays with phenylacetamide.

\section{RESULTS}

The strains of Pseudomonas putida A, P. cepacia and P. acidovorans listed in Table I grew on minimal agar plates with phenylacetamide as the nitrogen or carbon source. Pseudomonas cepacia grew vigorously on $\mathrm{S} / \mathrm{Ph}$ (succinate + phenylacetamide) plates after I to 2 days; the $P$. acidovorans strains grew less well, while $P$. putida A87 grew less well than $P$. cepacia $7 \mathrm{I} 6$ and the acetamide-negative strain $P$. putida A90. Pseudomonas aeruginosa mutant strain PAC377 grew rather better than the other species on $\mathrm{S} / \mathrm{Ph}$ plates.

It was thought possible that the rate-limiting step for growth of these pseudomonads on $\mathrm{S} / \mathrm{Ph}$ plates could be the rate of phenylacetamide hydrolysis. The specific amidase activities of cultures were compared after overnight growth in minimal salts medium in the presence and absence of acetamide and phenylacetamide. Amide transferase activity is directly related to the amide hydrolase activity of these enzymes (Betz \& Clarke, 1972; Clarke, 1972). Since the transferase assay is simpler and more convenient all the assays were done by the transferase method (Brammar \& Clarke, 1964) and results are expressed as values for amide transferase activity with hydroxylamine as the acceptor of the acyl group.

Table 2 shows that overnight growth of the wild-type strains of Pseudomonas aeruginosa PACI, $P$. putida A87, P. cepacia $7 \mathrm{I} 6$ and $P$. acidovorans $\mathrm{NCIB} 968 \mathrm{I}$ in minimal salts medium 
Table I. Strains of Pseudomonas species

Strain no.

Pseudomonas aeruginosa

PACI

PACI 28

PAC 377

Pseudomonas putida

A87, A56, A76, AI 54

A87CI

A $87 \mathrm{C} 3$

A90

Pseudomonas cepacia

716,727

$716 / 5$

Pseudomonas acidovorans

NCIB $9681 \dagger$

105
Acetamidase activity

\author{
Wild-type, Ind, $\mathrm{A}$ amidase \\ Con, $\mathrm{A}$ amidase \\ Con, $\mathrm{PhB}_{3}$ amidase*
}

Amide growth phenotype

$$
\begin{aligned}
& \mathrm{A}^{+} \mathrm{Ph}^{-} \\
& \mathrm{A}^{+} \mathrm{Ph}^{-} \\
& \mathrm{A}^{-} \mathrm{Ph}^{+}
\end{aligned}
$$

Wild-type, Ind
Con
Neg

$\mathrm{A}^{+} \mathrm{Ph}^{+}$

$\mathrm{A}^{+} \mathbf{P h}^{+}$

$\mathrm{A}^{-} \mathrm{Ph}^{+}$

$\mathrm{A}^{-} \mathrm{Ph}^{+}$

Wild-type, Ind

Neg

Wild-type, Ind

Wild-type, Ind
$\mathrm{A}^{+} \mathrm{Ph}^{+}$

A- $\mathrm{Ph}^{+}$

$\mathrm{A}^{+} \mathrm{Ph}^{+}$

$\mathrm{A}+\mathrm{Ph}^{+}$
Reference

Kelly \& Clarke (1960)

Brown \& Clarke (1970)

Betz \& Clarke (1972)

Stanier et al. (1966)

Clarke (1972)

This paper

Stanier et al. (1966)

Ballard et al. (1970)

This paper

Stanier et al. (1966)

* Mutant enzyme derived by mutation of Pseudomonas aeruginosa acetamidase.

$\uparrow$ Strain was derived from the same original culture as Pseudomonas acidovorans strain 14 of Stanier et al. (1966).

Abbreviations: Ind, inducible; Con, constitutive; Neg, negative for acetamide transferase; $\mathrm{A}^{\dagger}$, growth on minimal agar plates with $0.3 \%(\mathrm{w} / \mathrm{v})$ acetamide as carbon and nitrogen source; $\mathrm{Ph}^{+}$, growth on minimal agar plates with succinate $\mathrm{I} \cdot 0 \%(\mathrm{w} / \mathrm{v})+$ phenylacetamide $0 \cdot \mathrm{I} \%(\mathrm{w} / \mathrm{v})$.

\section{Table 2. Acetamide and phenylacetamide transferase activities}

Cultures were grown overnight in minimal salts medium containing acetamide or phenylacetamide to induce enzyme synthesis and in pyruvate or lactate medium as uninduced control. Lactamide was added to a lactate medium for Pseudomonas putida A90 in an attempt to elicit acetamidase synthesis.

Strain no.

Pseudomonas aeruginosa

PACI

PACI 28

PAC 377

Pseudomonas putida

A87

A90

Pseudomonas cepacia 716

Pseudomonas acidovorans

NCIB $968 \mathrm{I}$
Carbon source

Acetamide

Pyruvate

Pyruvate

Pyruvate

Acetamide

Lactate Phenylacetamide

Lactate Lactamide

Phenylacetamide

Pyruvate

Acetamide

Phenylacetamide

Lactate

Acetamide

Phenylacetamide
Specific activity $\mu \mathrm{mol}$ acyl hydroxamate formed/mg dry wt bacteria/min

Acetamide Phenylacetamide

$\begin{array}{lr}15.8 & <0.01 \\ 30 & <0.01 \\ 0.01 & 0.26\end{array}$

$\begin{array}{rr}0.4 & <0.01 \\ 10.5 & <0.03 \\ 0.16 & 0.08 \\ <0.01 & <0.01 \\ <0.04 & 0.16\end{array}$

$\begin{array}{rr}0.07 & <0.01 \\ 6.9 & <0.01 \\ <0.01 & 0.08\end{array}$

0.09

$12 \cdot 7$

$<0.01$

$<0.01$

0.06

0.05 
containing acetamide induced a high level of acetamidase activity but that no phenylacetamidase activity was detected. Pseudomonas putida A9o does not grow on acetamide, and acetamidase was not induced when cultures were grown in lactate medium in the presence of lactamide, an amide which is a good inducer for acetamide-positive strains. Growth in the presence of phenylacetamide resulted in low but measurable phenylacetamidase activity in the wild-type strains $P$. putida A87 and A90, $P$. cepacia 716 and $P$. acidovorans NCIB 968r without any significant increase in the acetamidase activity over the basal level which may be reached in the absence of acetamide. The phenylacetamidase activities of the $P$. putida, $P$. cepacia and $P$. acidovorans strains were lower than those of the $P$. aeruginosa phenylacetamide-utilizing mutants grown under the same conditions (Betz \& Clarke, I972). However, these activities would be sufficient to account for the utilization of phenylacetamide by these strains.

\section{Growth and utilization of acetamide and phenylacetamide}

More detailed studies on the relationships between amidase activities and growth were carried out by measuring growth, amide disappearance and amidase synthesis in cultures incubated in minimal salts medium containing either acetamide $(50 \mathrm{~mm})$ or phenylacetamide $(6 \mathrm{~mm})$. The strains were grown overnight in the same medium as was then used in the experimental flasks, or in lactate (+ ammonium) salts medium if the test amide did not support growth. Prewarmed flasks of the test media were inoculated with the overnight cultures at $I /$ Ioth or $I / 20$ th of the medium volume. The flask cultures were incubated on a shaker and samples removed at intervals for measurements of growth and enzyme assays. Pseudomonas aeruginosa strains were grown at $37^{\circ} \mathrm{C}$ and $0.5 \%$ pyruvate was added to the phenylacetamide medium as the carbon source; the other strains were grown at $30{ }^{\circ} \mathrm{C}$ and phenylacetamide provided both the carbon and nitrogen sources for growth.

The Pseudomonas aeruginosa mutant PAC377 resembled the wild-type $P$. putida A90 in that it grew on succinate + phenylacetamide plates but not on acetamide. Fig. I and 2 show that no growth occurred, and no disappearance of acetamide could be detected, when these strains were incubated for $8 \mathrm{~h}$ in acetamide medium, confirming that they could both be regarded as acetamide-negative strains. Pseudomonas putida A90 grew rather slowly in the phenylacetamide medium during the $8 \mathrm{~h}$ incubation period with a mean generation time of $210 \mathrm{~min}$; there was low but significant increase in phenylacetamidase activity and phenylacetamide was hydrolysed slowly. After $24 \mathrm{~h}$ the optical density of the culture $\left(E_{670 \mathrm{um}}\right)$ had reached a value of 0.7 equivalent to about $10^{9}$ bacteria $/ \mathrm{ml}$ and the phenylacetamide was completely hydrolysed. These results suggest that the rate of growth of $P$. putida A90 in phenylacetamide medium was determined by the rate of phenylacetamidase synthesis.

Pseudomonas aeruginosa PAC377 is constitutive for amidase synthesis but there was a lag before a significant level of phenylacetamidase could be detected. The mean generation time in pyruvate and phenylacetamide medium was $180 \mathrm{~min}$ and phenylacetamide began to disappear from the medium at an early stage. Since there was no lag in growth or phenylacetamide hydrolysis it is possible that the apparent lag in amidase synthesis was due to the insensitivity of the assay with the dilute bacterial suspensions at the early stages of growth. Since the mean generation time of the parent strain PACI in pyruvate medium with ammonia as the nitrogen source is $70 \mathrm{~min}$, the rate-limiting step for growth in the pyruvate + phenylacetamide medium may be the rate of phenylacetamide hydrolysis.

Another factor which could affect the growth rate in phenylacetamide media is the rate of uptake of phenylacetamide from the culture medium. This was not tested directly, but when enzyme assays of whole organisms and extracts of any of the strains were compared, there was no instance in which the specific phenylacetamidase activity of an extract was greater 

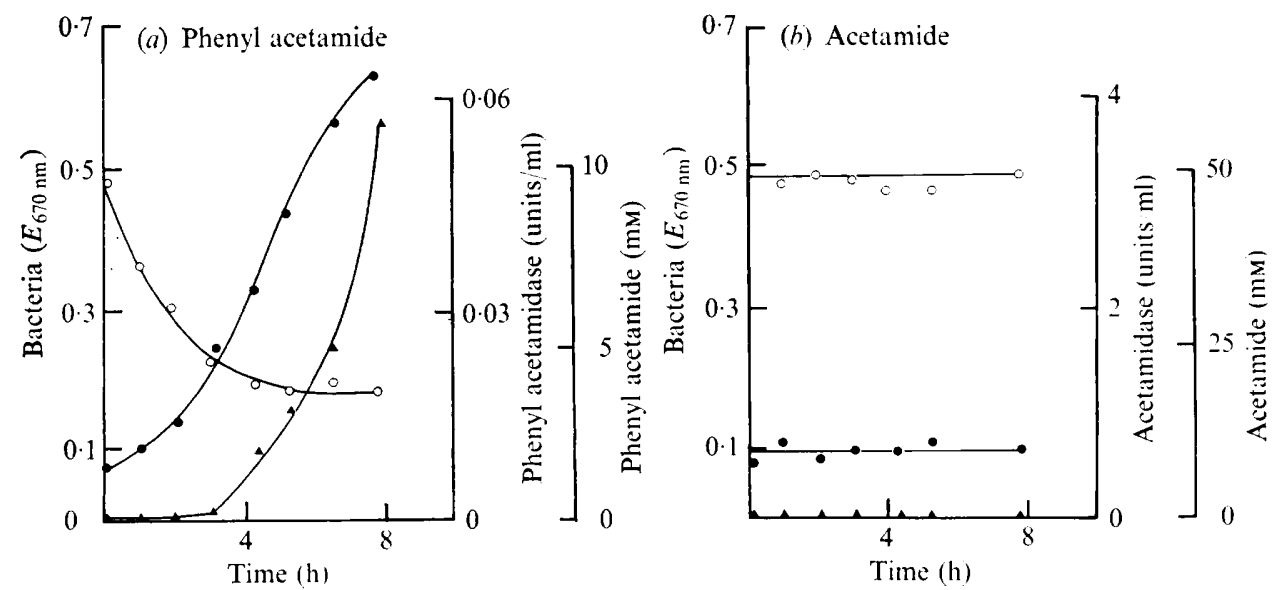

Fig. I. Growth and utilization of amides by Pseudomonas aeruginosa PAC377. - Bacterial growth $\left(E_{670 \mathrm{~mm}}\right) ; \bigcirc-\bigcirc,(a)$ phenylacetamide concentration $(\mathrm{mM}),(b)$ acetamide concentration $(\mathrm{mM})$; $\Delta-\boldsymbol{\Lambda},(a)$ phenylacetamidase activity (units $/ \mathrm{ml}$ ), $(b)$ acetamidase activity (units $/ \mathrm{ml}$ ).
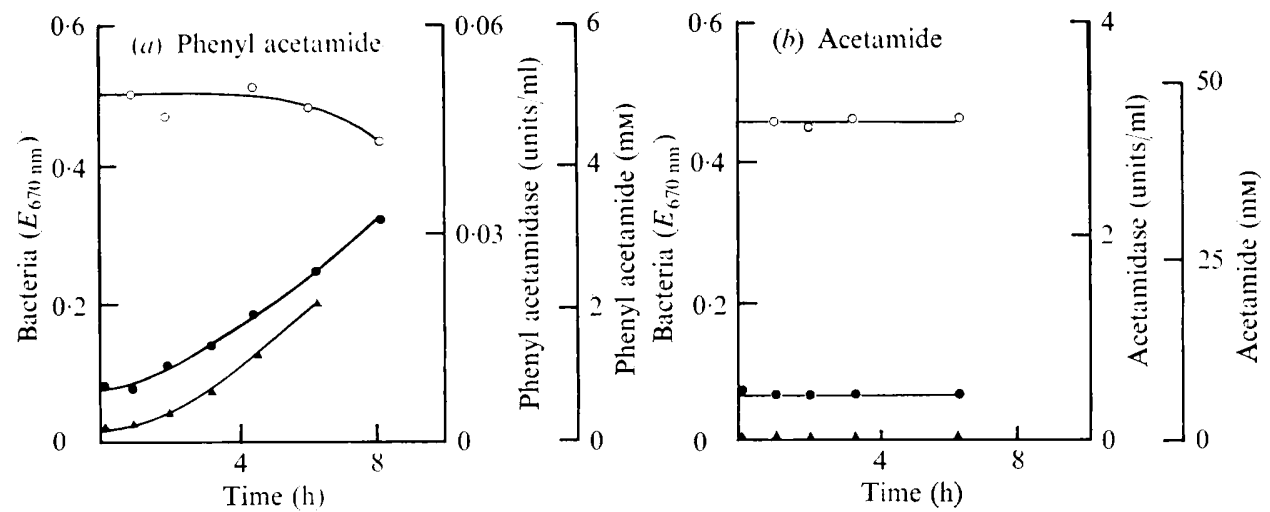

Fig. 2. Growth and utilization of amides by Pseudomonas putida A90. - Bacterial growth $\left(E_{670 \mathrm{~nm}}\right) ; \bigcirc-\bigcirc,(a)$ phenylacetamide concentration $(\mathrm{mm}),(b)$ acetamide concentration $(\mathrm{mM}) ; \boldsymbol{\Delta}-\boldsymbol{\Lambda}$, (a) phenylacetamidase activity (units/ml), (b) acetamidase activity (units/ml).

than that of the suspension of organisms from which it was derived. Pseudomonas aeruginosa is known to have a constitutive acetamide permease (Brammar, McFarlane \& Clarke, I966).

Pseudomonas cepacia 716 grew well on both acetamide and succinate + phenylacetamide plates and the amidase activities of overnight cultures grown in various media (Table 2 ) had suggested that growth on phenylacetamide might be due to a second amidase with a different specificity from the aliphatic amidase previously described (Clarke, 1972). Cultures grew well in minimal salt medium with either acetamide or phenylacetamide as the carbon and nitrogen source for growth with a mean generation time of about $165 \mathrm{~min}$ in both media. Fig. 3 shows that acetamide began to disappear from the culture early during growth and phenylacetamide after about 3 h. The wild-type strain of $P$. aeruginosa PACI grows on acetamide with a mean generation time of about $50 \mathrm{~min}$ and in a comparable experiment acetamide, which was present at an initial concentration of $50 \mathrm{~mm}$, disappeared much more rapidly and was completely lost from the culture medium in about I h (Brammar \& Clarke, 1964). Fig. 3 

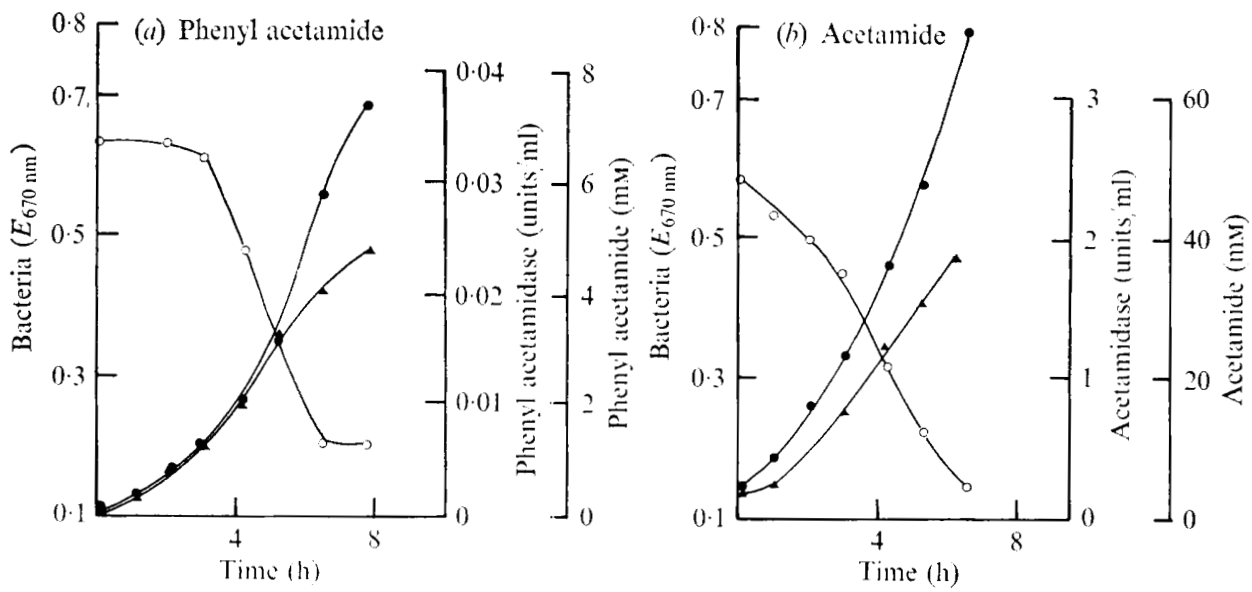

Fig. 3. Growth and utilization of amides by Pseudomonas cepacia 716 . -1 , Bacterial growth $\left(E_{670 \mathrm{~nm}}\right) ; \bigcirc-\bigcirc,(a)$ phenylacetamide concentration $(\mathrm{mM}),(b)$ acetamide concentration $(\mathrm{mm})$; $\boldsymbol{\Delta}-\boldsymbol{\Lambda},(a)$ phenylacetamidase activity (units $\mathrm{ml}$ ), (b) acetamidase activity (units/ml).
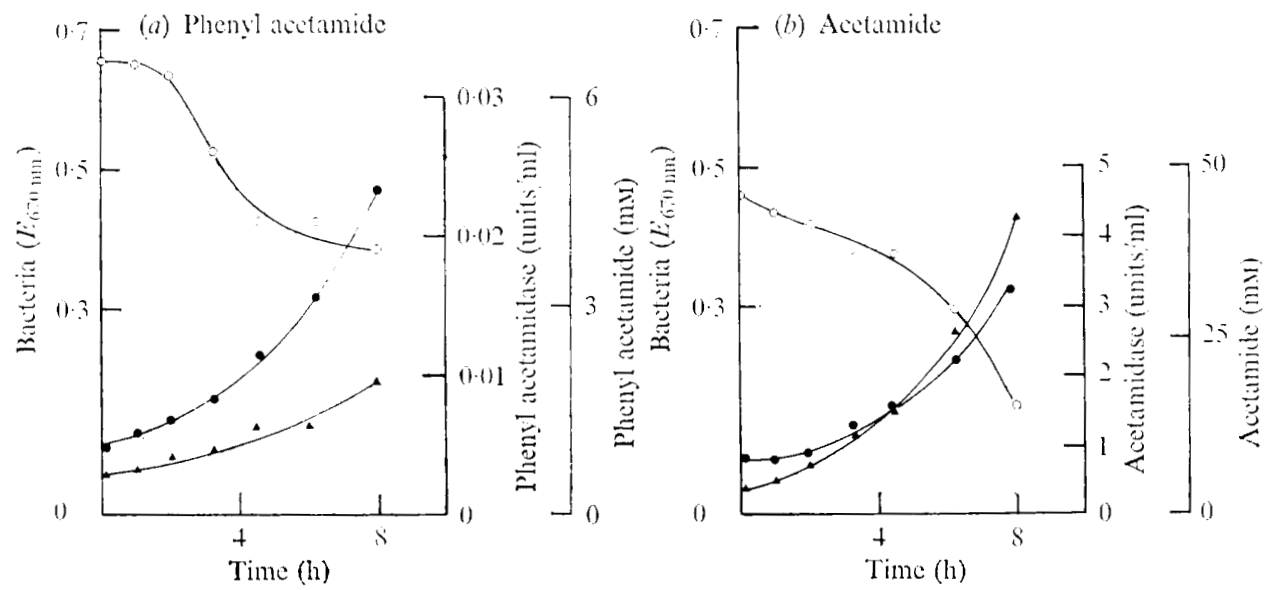

Fig. 4. Growth and utilization of amides by Pseudomonas acidovorans NCiB 968I. - Bacterial growth $\left(E_{670 n \mathrm{~m})}\right) ;-\mathrm{C},(a)$ phenylacetamide concentration $(\mathrm{mM}),(b)$ acetamide concentration $(\mathrm{mM}) ; \boldsymbol{\Delta}-\boldsymbol{\Delta},(a)$ phenylacetamidase activity (units $/ \mathrm{ml}$ ) (b) acetamidase activity (units $/ \mathrm{ml}$ ).

shows that the specific phenylacetamidase activities of the $P$. cepacia cultures throughout growth were $\mathrm{I} \%$ or less of the acetamidase specific activities of the cultures growing on acetamide.

Pseudomonas acidovorans NCIB 968I also grew in both acetamide and phenylacetamide medium but for this strain the rate of growth on acetamide was slightly lower than that in the phenylacetamide medium (Fig. 4). The acetamidase activity of the culture was reasonably high and it is possible that the rate-limiting step for growth of this strain on acetamide is not the rate of acetamide hydrolysis but the rate of acetate metabolism. The phenylacetamidase activity of the culture growing in the phenylacetamide medium was very low but increased during the experimental period and after $24 \mathrm{~h}$ all the phenylacetamide had disappeared from the medium. 
Table 3. Oxidation of phenylacetamide by washed suspensions of Pseudomonas spp.

Cultures were grown overnight in minimal salts medium with the compounds listed below. The bacteria were washed and resuspended in tris buffer, $\mathrm{pH} 7 \cdot 2,0.1 \mathrm{M}$. Manometer flasks contained I to $2 \mathrm{mg}$ dry wt bacteria, in $3 \mathrm{ml}$ buffer, with $0.2 \mathrm{ml} 30 \%(\mathrm{w} / \mathrm{v}) \mathrm{KOH}$ in the centre well. Phenylacetamide $(80 \mu \mathrm{mol})$ was added from the sidearm as a suspension in distilled water.

Strain no.

Pseudomonas aeruginosa $\mathrm{PAC} 377$

Pseudomonas putida A90

A87

Pseudomonas cepacia 716

Pseudomonas acidovorans NCIB 968I $\mu 1 \mathrm{O}_{2}$ uptake/h/mg dry wt

Additions to medium

Lactate/phenylacetamide

Phenylacetamide

Phenylacetamide

Lactate

Phenylacetamide/lactate

Phenylacetamide

Lactate

$\begin{array}{cc}\text { Phenylacetamide } & \text { Endogenous } \\ 91^{*} & 91^{*} \\ 270 & 39 \\ 121 & 32.5 \\ 28.5 & 20.5 \\ 62.5 & 25.9 \\ 150 & 26 \\ 19.3 & 16.2\end{array}$

* Values obtained with $\mathrm{I} \mathrm{ml}$ of suspension about $3 \mathrm{mg}$ dry wt bacteria.

Utilization of phenylacetamide as a carbon source for growth

Pseudomonas putida A90, $P$. cepacia $7 \mathrm{I} 6$ and $P$. acidovorans $968 \mathrm{I}$ were able to utilize phenylacetamide as a carbon as well as a nitrogen source. Phenylacetate is metabolized by pseudomonads by a $m$-cleavage pathway and, as is the case for other aromatic pathways investigated, the enzymes are mostly inducible (Dagley, 197I). Growth on phenylacetamide ought to induce the enzymes necessary for phenylacetamide oxidation as well as hydrolysis. Table 3 compares the rates of oxidation by washed suspensions of cultures grown in lactate $(0.5 \%)$ or phenylacetamide $(0.1 \%)$ medium; there were no significant differences between the values obtained for endogenous oxygen uptake and for oxygen uptake in the presence of phenylacetamide for any of the cultures grown in lactate medium. These cultures therefore lacked both phenylacetamidase and the oxidizing system. The $Q_{\mathrm{O}_{2}}$ (uncorrected) for phenylacetamide of the phenylacetamide-grown suspension of $P$. putida A87 was 121 , for A90 it was 270 and for $P$. acidovorans NCIB $968 \mathrm{I}$ it was $\mathrm{I} 50$. The endogenous values for these strains ranged in these experiments from 26 to 39. The $P$. cepacia 7 I 6 suspensions tended to give higher endogenous values but the phenylacetamide-grown culture had a lower endogenous rate of oxidation than the lactate-grown culture and the rate of phenylacetamide oxatidion was more than twice the endogenous rate. On the other hand a washed suspension of the phenylacetamide-utilizing mutant of $P$. aeruginosa, grown in lactate + phenylacetamide medium, was unable to oxidize phenylacetamide. Phenylacetate accumulated in the Warburg flasks in which the phenylacetamide-grown suspensions of $P$. aeruginos $a$ PAC 377 had been incubated with phenylacetamide, whereas only trace amounts were detected in comparable flasks for the other Pseudomonas spp. The rate of oxygen uptake was linear for at least $60 \mathrm{~min}$ with washed suspensions of all these strains.

\section{Growth characteristics of acetamide negative strains}

Pseudomonas putida A9o is a naturally occurring acetamide-negative strain, and the results we had obtained suggested that it produced a single amidase which could attack phenylacetamide but not acetamide. Strain A87 grew on both acetamide and phenylacetamide but the very low activities of the phenylacetamidases of these cultures made it difficult to be 

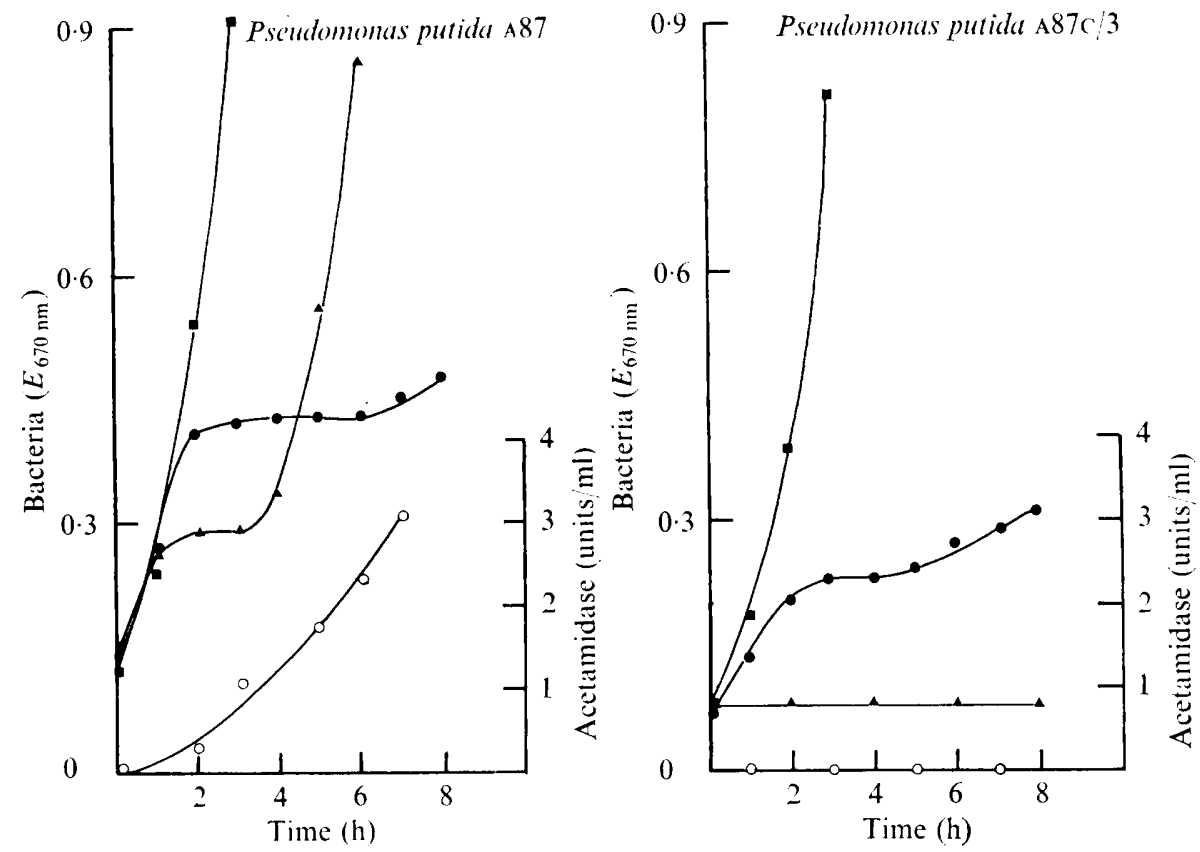

Fig. 5. Growth of Pseudomonas putida A87 and the acetamide negative mutant A87C/3 in minimal salt media measured as $E_{670 m m}$. Additions: $-\mathbf{-}, 0.5 \%$ sodium lactate $+0.1 \%\left(\mathrm{NH}_{4}\right)_{2} \mathrm{SO}_{4} ; \boldsymbol{\Delta}-\boldsymbol{\Delta}$, $0.5 \%$ acetamide; $-0.5 \%$ sodium lactate $+0.1 \%$ phenylacetanide. $\mathrm{C}-\mathrm{O}$, Acetamidase activity (units/ml).

certain that growth on phenylacetamide was not due to trace activity of the aliphatic amidase previously described. A number of acetamide-negative mutants was isolated from a constitutive mutant $\mathrm{A} 87 \mathrm{C}$, and all these were found to grow as well as the parent strain on pyruvate + phenylacetamide. Pyruvate was used instead of succinate to increase sensitivity since it gives less catabolite-repression than succinate.

One of the Pseudomonas putida mutants was examined in more detail. The growth of the acetamide-negative mutant $\mathrm{A} 87 \mathrm{C} / 3$ was compared with that of strain $\mathrm{A} 87$ in minimal salts media containing as carbon and nitrogen sources the following: lactate $(0.5 \%)+$ ammonium sulphate $(0.1 \%)$; acetamide $(0.5 \%)$; lactate $(0.5 \%)$ t phenylacetamide $(0 . \mathrm{I} \%)$. The flasks were inoculated with $\mathrm{I} / 2$ oth of the medium volume of overnight cultures grown in lactate medium, lactate being another carbon source which does not produce severe catabolite repression of amidase synthesis. Fig. 5 shows that both strains grew rapidly and without lag in the lactate medium, showing that the mutant was not impaired in normal growth on lactate. A87 grew well on acetamide exhibiting a typical growth diauxie. Acetamidase began to be synthesized during the diauxic lag and reached an appreciable rate of synthesis about $\mathrm{I} h$ later. There was a more marked growth diauxie in the phenylacetamide medium for $\mathrm{A} 87$ and growth on phenylacetamide was not detected until $6 \mathrm{~h}$ after inoculation.

The mutant $\mathrm{A} 87 \mathrm{C} / 3$ did not grow at all on acetamide and no acetamidase activity was detected. Growth of $\mathrm{A} 87 \mathrm{C} / 3$ on phenylacetamide was comparable with that of strain $\mathrm{A} 87$. The volumes of the samples removed during growth were too small for phenylacetamidase activity to be detected during this period of growth. After $24 \mathrm{~h}$, phenylacetamidase activity was detected in the cultures grown in the lactate + phenylacetamide medium but in none of the other cultures of $\mathrm{A} 87$ or $\mathrm{A} 87 \mathrm{C} / 3$. 

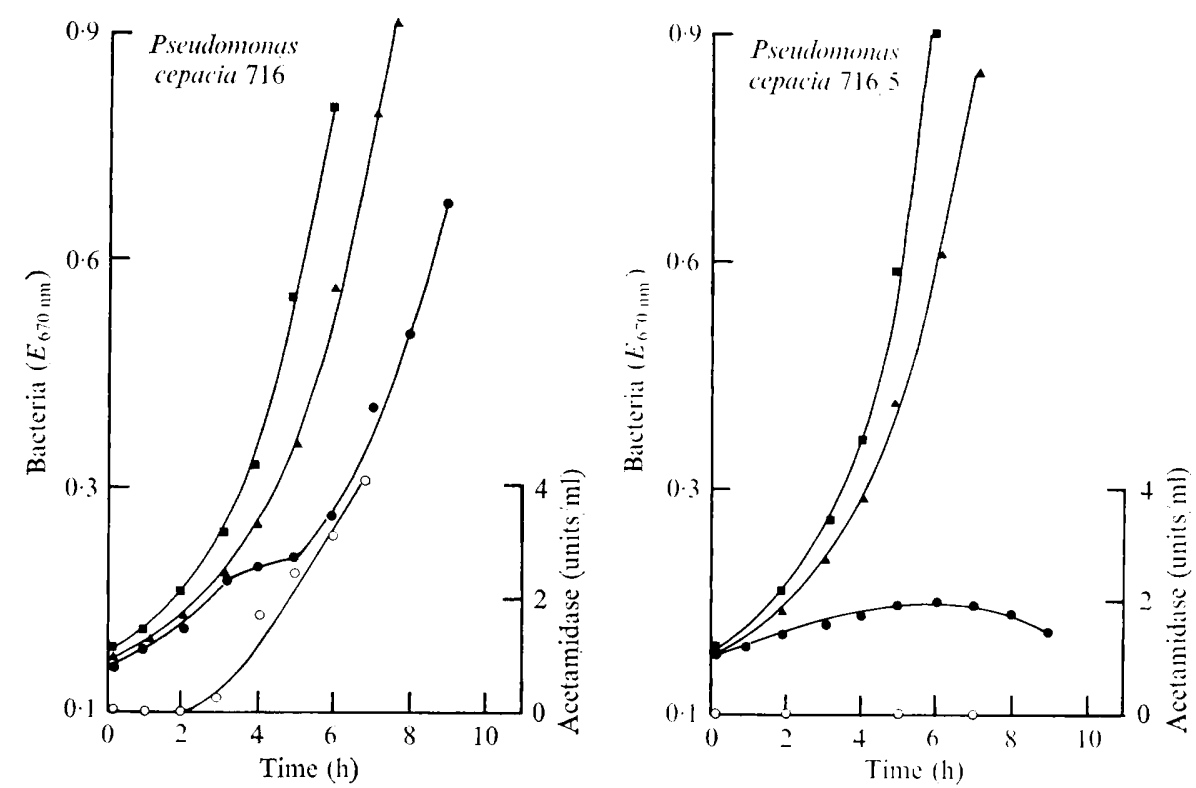

Fig. 6. Growth of Pseudomonas cepacia 716 and the acetamide-negative mutant $716 / 5$ in minimal salt media measured as $E_{670 \mathrm{~nm}}$. Additions: $\mathbf{\square}-0.5 \%$ sodium lactate $+0.1 \%\left(\mathrm{NH}_{4}\right) \mathrm{SO}_{4}$; $-0,0.5 \%$ acetamide; $\boldsymbol{\Delta}-\boldsymbol{\Delta}, 0.5 \%$ sodium lactate $+0.1 \%$ phenylacetamide. $\mathrm{O}-\mathrm{O}$, Acetamidase activity (units/ml).

Cultures of $\mathrm{A} 87$ and $\mathrm{A} 87 \mathrm{C} / 3$ were grown in $100 \mathrm{ml}$ volumes of lactate and lactate + lactamide medium for the preparation of concentrated cell-free extracts. The extract from $\mathrm{A} 87$ grown in the lactate + lactamide medium had a very high acetamidase activity and gave a very distinct band in the immunodiffusion reaction carried out against antiserum to Pseudomonas aeruginosa A amidase (Clarke, 1972). The extract from $\mathrm{A} 87 \mathrm{C} / 3$ had no acetamidase activity and gave no cross-reaction in the immunodiffusion test. Growth of $P$. putida A87 on phenylacetamide thus did not depend on the presence of the aliphatic amidase but was due to a separate enzyme.

Similar experiments were carried out with acetamide-negative mutants isolated from Pseudomonas cepacia 716. Mutants which had lost the ability to grow on acetamide plates still grew very well on phenylacetamide plates and on pyruvate + phenylacetamide plates. Fig. 6 compares the growth of one of these mutants $716 / 5$ with its parent strain in $(a)$ lactate + ammonium salts; $(b)$ acetamide; and (c) lactate + phenylacetamide media. Both strains grew rapidly without lag in the lactate medium. In contrast to the previous experiment with $P$. putida, growth of the two strains of $P$. cepacia on the lactate + phenylacetamide medium occurred after only a short lag and soon reached approximately the same rate as in the lactate + ammonium salt medium. Strain 716 had been observed to grow much more vigorously than $\mathrm{A} 87$ on pyruvate + phenylacetamide plates and produced higher levels of phenylacetamidase during growth on phenylacetamide than $P$. putida A90 and $P$. acidovorans NCIB 968I. In this experiment it appears that the growth rate is not limited by the rate of phenylacetamide hydrolysis by either of these strains. Growth of strain 7I 6 in the acetamide medium showed a typical diauxic lag and the aliphatic amidase began to be synthesized during this period. The mutant 7 16/5 did not produce any aliphatic amidase and the slight increase in bacterial density was probably due to slight growth on the residual nutrients of the 
Table 4. Amide growth range of Pseudomonas strains

Growth was recorded after 4 days at $30^{\circ} \mathrm{C}$. Composition of media as in Methods section.

$\begin{array}{ccccccc}\text { Strain no. } & \text { A } & \text { B } & \text { V } & \text { H } & \text { Pyr/Ph } & \text { Ph } \\ \text { Pseudomonas aeruginosa } \mathrm{PACI} & + & - & - & - & - & - \\ \text { PAC377 } & - & + & + & + & + & - \\ \text { Pseudomonas putida } \text { A87 } & + & + & - & - & + & + \\ \text { A87C/3 } & - & - & - & - & + & + \\ \text { A90 } & - & - & - & - & + & + \\ \text { Pseudomonas cepacia } 716 & + & - & + & + & + & + \\ 716 / 5 & - & - & + & + & + & + \\ \text { Pseudomonas acidovorans } \mathrm{NCIB} 968 \mathrm{I} & + & + & - & - & + & +\end{array}$

inoculation medium. It was clear that this mutant was not using the aliphatic amidase to metabolize phenylacetamide.

We have examined the growth of the wild-type strains and the mutants on minimal agar plates with valeramide and hexanoamide as carbon and nitrogen sources as well as the lower aliphatic amides and phenylacetamide (Table 4). The Pseudomonas putida mutant A87C/3 was identical in amide growth range with the wild-type strain 490 and grew on phenylacetamide but not on valeramide and hexoanoamide. Pseudomonas cepacia $7 \mathrm{I} 6$ has a wider amide growth range than any of the other species and this, together with the absence of growth on butyramide, had suggested that there were at least two amidases with quite different substrate specificities. The acetamide-negative mutant $716 / 5$ grew on valeramide and hexoanoamide as well as phenylacetamide. It is possible that the enzyme we have been examining as a phenylacetamidase has a broad substrate specificity which allows it to attack this group of amides. If we compare $P$. cepacia $716 / 5$ with the $P$. aeruginosa mutant $\mathrm{PAC} 377$, the amide growth range is similar, although the $P$. aeruginosa mutant is also able to grow on butyramide. PAC 377 produces a single amidase with this broad substrate specificity (Betz \& Clarke, 1972) and the $P$. cepacia phenylacetamidase may be a similar enzyme although it cannot be completely ruled out from these findings that a third amidase is produced which attacks valeramide and hexoanoamide.

\section{DISC.USSION}

The amide growth patterns of the four species of Pseudomonas studied had suggested that, while Pseudomonas aeruginosa strains produce an aliphatic amidase which acts only on aliphatic amides containing up to four carbon atoms the other species might produce in addition another amidase which was capable of attacking higher molecular weight amides. The growth characteristics of the wild-type strains in media in which either acetamide or phenylacetamide provided the nitrogen or carbon source for growth, together with the genetic evidence that mutants totally lacking acetamidase activity were still able to grow on phenylacetamide, have confirmed this view. We suggest that, for those species with both activities, the first enzyme should continue to be called the aliphatic amidase and that, for the time being, the second enzyme should be referred to as the phenylacetamidase. The wildtype $P$. aeruginosa strains can therefore be described as producing only an aliphatic amidase, while $P$. putida A strains, such as A9o, produce only the phenylacetamidase, and $P$. putida A87 and $P$. cepacia 716 produce both an aliphatic amidase and a phenylacetamidase.

The relative specific activities of the two amidases produced by the wild-type strains during growth on acetamide and phenylacetamide respectively, are in the ratio of 100: I when 
measured as specific activities for acetamide and phenylacetamide in the transferase reaction. It might be thought the hydrolase activities of the phenylacetamidases would be higher than the transferase activities but this has been tested with the Pseudomonas aeruginosa mutant phenylacetamidases and with cell-free extracts of the other species and the hydrolase and transferase specific activities were similar. The phenylacetamidase of P. cepacia 716 was the most active of the enzymes measured and in one experiment a hydrolase value of $0.06 \mu \mathrm{mol}$ of ammonia $/ \mathrm{mg}$ protein $/ \mathrm{min}$ was obtained with a cell-free extract of a culture grown in phenylacetamide medium, which was comparable with the specific activities measured by the transferase reaction for cultures grown in the same medium.

The very low specific activities of the phenylacetamidases appear to be sufficient to support growth of the strains described, although in some instances, particularly for Pseudomonas putida $\mathrm{A} 87$, it is suggested that the rate of hydrolysis of phenylacetamide is the rate-limiting step for growth in media which contain this compound as the nitrogen source. Since the phenylacetamidases are inducible it is possible that higher specific activities may be obtained by the isolation of constitutive strains. However, the phenylacetamide-utilizing mutants of P. aeruginosa (Betz \& Clarke, 1972), which grow very well on phenylacetamide and are constitutive for amidase synthesis, also have very low specific activities for phenylacetamide. This may not be a fair comparison since these are all mutant enzymes obtained after successive mutations of the aliphatic amidase whereas the $P$. putida, $P$. cepacia and $P$. acidovorans phenylacetamidases have evolved by natural selection.

It is possible that the two types of amidase have been derived from a common ancestral enzyme. If the phenylacetamidases can be obtained in sufficient quantity by the isolation of derepressed mutants it would be interesting to compare them with the aliphatic amidases produced by these species.

\section{REFERENCES}

Ballard, R. W., Palleroni, N. J., Doudoroff, M. \& Stanier, R. Y. (i970). Taxonomy of the aerobic pseudomonads: Pseudomonas cepacia, P. marginata, $P$. allicola and P. caryophylli. Journal of General Microbiology 6o, 119-214.

Betz, J. L. \& Clarke, P. H. (I972). Selective evolution of phenylacetamide-utilizing strains of Pseudomonas aeruginosa. Journal of General Microbiology 73, 161-174.

Brammar, W. J. \& Clarke, P. H. (1964). Induction and repression of Pseudomonas aeruginosa amidase. Journal of General Microbiology 37, 307-319.

Brammar, W. J., Clarke, P. H. \& Skinner, A. J. (1967). Biochemical and genetic studies with regulator mutants of the Pseudomonas aeruginosa 8602 amidase system. Journal of General Microbiology 47, 87-102.

Brammar, W. J., McFarlane, N. D. \& Clarke, P. H. (1966). The uptake of aliphatic amides by Pseudomonas aeruginosa. Journal of General Microbiology 44, 303-309.

Brown, J. E. \& Clarke, P. H. (1970). Mutations in a regulator gene allowing Pseudomonas aeruginosa 8602 to grow on butyramide. Journal of General Microbiology 64, 329-342.

Clarke, P. H. (1972). Biochemical and immunological comparison of aliphatic amidases produced by Pseudomonas species. Journal of General Microbiology 7I, 24I-257.

Clarke, P. H. \& TATA, R. (I973). Isolation of amidase-negative mutants of Pseudomonas aeruginosa by a positive selection method using an acetamide analogue. Journal of General Microbiology $\mathbf{7 5}$, $231-234$.

Dagley, S. (1971). Catabolism of aromatic compounds by micro-organisms. Advances in Microbiol Physio$\log y 6, \mathrm{I}-46$.

Kelly, M. \& Clarke, P. H. (1962). An inducible amidase produced by a strain of Pseudomonas aeruginosa. Journal of General Microbiology 27, 305-316.

Stanier, R. Y., Palleroni, N. J. \& Doudoroff, M. ( 1966). The aerobic pseudomonads; a taxonomic study Journal of General Microbiology 43, 159-27I. 